\title{
Blood pressure estimation from photoplethysmogram and electrocardiogram signals using machine learning
}

Yang, S, Zaki, W.S.W, Morgan, S.P, Cho, S.-Y, Correia, R, Wen, L., Zhang, Y

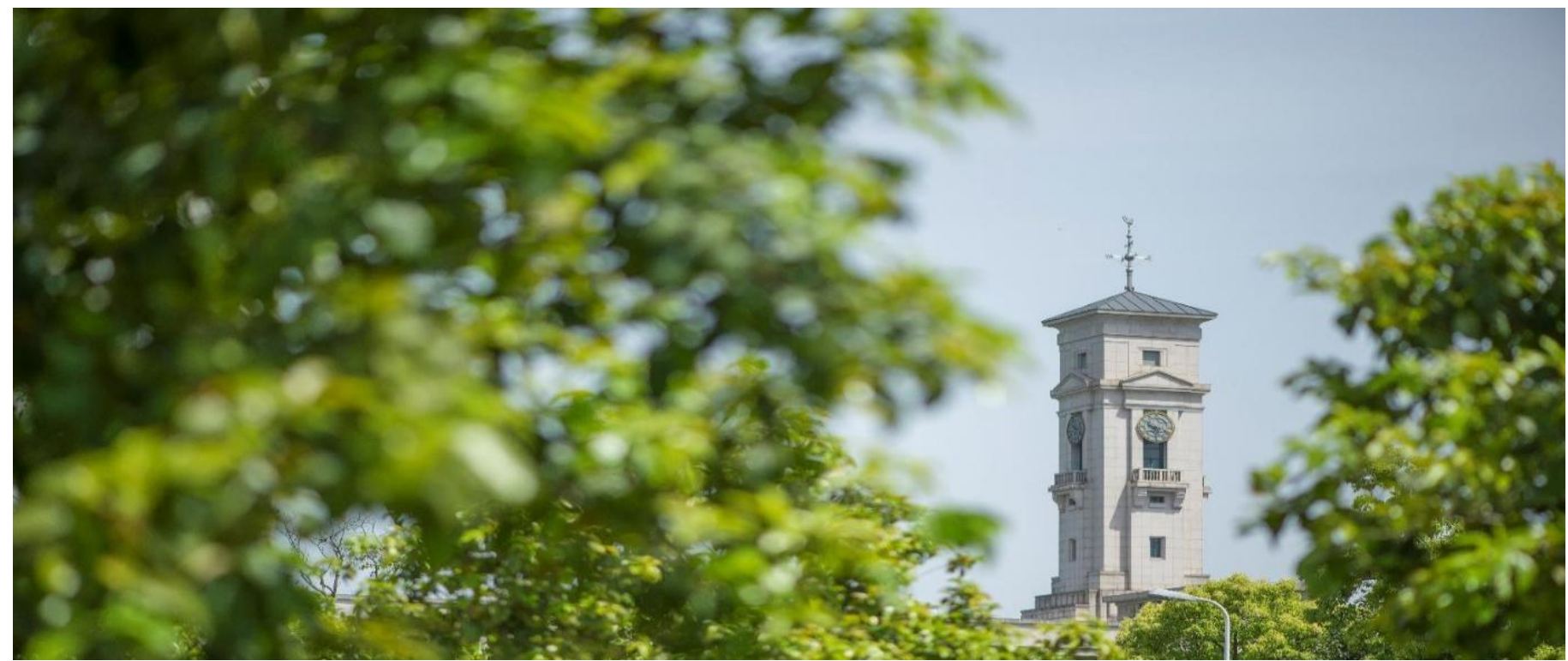


University of Nottingham Ningbo China, 199 Taikang East Road, Ningbo, 315100, China

First published 2018

This work is made available under the terms of the Creative Commons Attribution 4.0 International License:

http://creativecommons.org/licenses/by/4.0

The work is licenced to the University of Nottingham Ningbo China under the Global University Publication Licence:

https://www.nottingham.edu.cn/en/library/documents/researchsupport/global-university-publications-licence.pdf 


\title{
BLOOD PRESSURE ESTIMATION FROM
} PHOTOPLETHYSMOGRAM AND ELECTROCARDIOGRAM SIGNALS USING MACHINE LEARNING

\author{
SenYang ${ }^{1}$, Wan SW Zaki ${ }^{2}$, Stephen P.Morgan ${ }^{2}$, Siu-Yeung Cho ${ }^{1}$, Ricardo Correia ${ }^{2}$, \\ Long Wen ${ }^{3}$, Yaping Zhang \\ ${ }^{1}$ International Doctoral Innovation Centre, University of Nottingham Ningbo China, 199 Taikang East Road, \\ Ningbo, China \\ ${ }^{2}$ Optics and Photonics Research Group, University of Nottingham, Nottingham, United Kingdom \\ ${ }^{3}$ School of Economics, University of Nottingham Ningbo China, 199 Taikang East Road, Ningbo, China \\ *Yaping.ZHANG@nottingham.edu.cn
}

\begin{abstract}
Keywords: BLOOD PRESSURE, PHOTOPLETHYSMOGRAM (PPG), ELECTROCARDIOGRAM (ECG), FEATURES
\end{abstract}

\begin{abstract}
Blood pressure measurement is a significant part of preventive healthcare and has been widely used in clinical risk and disease management. However, conventional measurement does not provide continuous monitoring and sometimes is inconvenient with a cuff. In addition to the traditional cuff-based blood pressure measurement methods, some researchers have developed various cuff-less and noninvasive blood pressure monitoring methods based on Pulse Transit Time (PTT). Some emerging methods have employed features of either photoplethysmogram (PPG) or electrocardiogram (ECG) signals, although no studies to our knowledge have employed the combined features from both PPG and ECG signals. Therefore this study aims to investigate the performance of a predictive, machine learning blood pressure monitoring system using both PPG and ECG signals. It validates that the employment of the combination of PPG and ECG signals has improved the accuracy of the blood pressure estimation, compared with previously reported results based on PPG signal only.
\end{abstract}

\section{Introduction}

Blood pressure is an important indicator in healthcare. It has been widely recognized as a useful metric for the

improvement of survival rates of patients and the prevention of cardiovascular diseases. There are two main reasons for the increased attention towards the blood pressure monitoring. Firstly, there is an increased need for continuous blood pressure measurement [1], because hypertension is dangerous and its detection rate is low, particularly where there is a resource scarcity $[2,3]$. Secondly, modern science and innovative technologies have brought about a revolution in many interdisciplinary research areas related to healthcare, such as miniaturized wearable devices, artificial intelligence, and smart mobile phones for medical care purposes [4]. These factors have promoted the development and adoption of ubiquitous blood pressure measurement and monitoring.
Hypertension is called the 'silent killer' because most hypertension patients are not aware of their condition or the organ damage it causes [5]. Therefore, continuous blood pressure measurement is very important in modern medicine. Traditional measurement methods can be divided into invasive and non-invasive methods. Invasive blood pressure measurement includes catheterization; non-invasive methods include auscultation, oscillometry, volume clamping, and tonometry [6]. Invasive methods include significant drawbacks such as vein collapse in the elderly and secondary infections, while non-invasive methods require cuffs or expensive hardware. All of these methods depend on manual operation. Many researchers, therefore, are trying to find noninvasive, cuff-less and continuous ways to predict blood pressure values.

One possible non-invasive, cuff-less and continuous way to access the blood pressure is to measure the Pulse Transit Time (PTT) by calculating the time difference between the peaks of electrocardiogram (ECG) and photoplethysmogram (PPG) signals within one heartbeat. The PTT method is principled on the theory that the velocity of pulse waves propagating along the arterial tree depends on the value of blood pressure [7]. Once processed, PTT data displays an inverse relationship with blood pressure values. Various approximations of this relationship have been proposed, such as linear, logarithmic and exponential [8]. In addition to the PTT methods, previously reported studies have also tried to extract features from either PPG or ECG signal to predict blood pressure [9-15]. However, to the best of our knowledge, there is no report employing features from both PPG and ECG signals.

This study aims to explore whether the combination of PTT with both PPG and ECG features can improve the accuracy of the prediction of blood pressure, compared with a study based on the combination of PTT with PPG features only [16]. 


\section{Methodology}

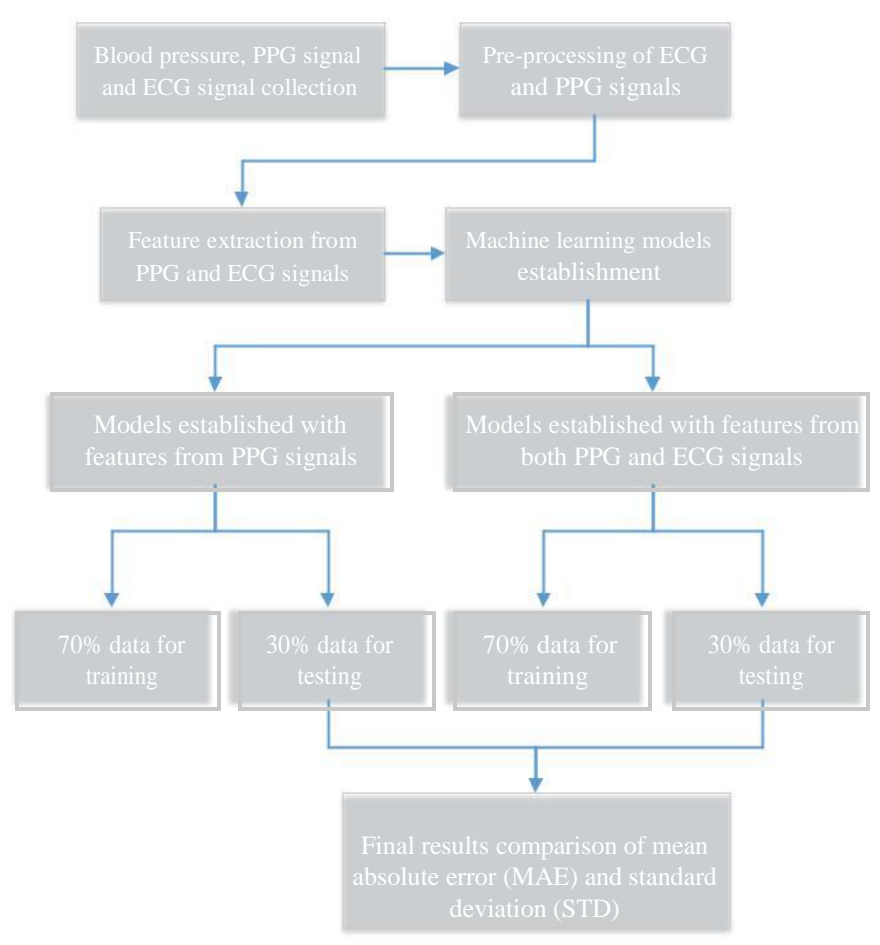

Fig. 1 Flowchart of the proposed approach for blood pressure estimation using features from both ECG and PPG signals

The implemented flowchart is shown in Fig.1, containing two main phases - data collection and signal analysis. In the feature extraction phase, both time and frequency domain features from both ECG and PPG signals were extracted.

After that, the datasets were divided into two groups, with $70 \% \quad b$ for training and $30 \%$ for testing. Finally, the projected blood pressure values were compared with conventional measurement readings, both with and without the inclusion of ECG features.

\subsection{Data collection}

Three types of data were collected in the lab simultaneously: ECG signal, PPG signal and sphygmomanometer blood pressure values. ECG signal was collected from surface electrodes adhered to subjects' skin, meanwhile, PPG signal was measured from a NeXus-10 Mark II biofeedback monitor. The sampling frequency for the data acquisition was 256 samples/second. The length of the recordings ranged from 14 seconds to more than 1 minute. In the experiment, 137 datasets were collected from 14 people under various scenarios after different levels of exercise. However, a few datasets were invalid as they either failed to record certain signals (e.g. missing certain blood pressure values) or included outliers. After screening the results, 129 datasets were reserved for next step of signal pre-processing. These datasets contained complete ECG, PPG, and systolic and diastolic blood pressure values.
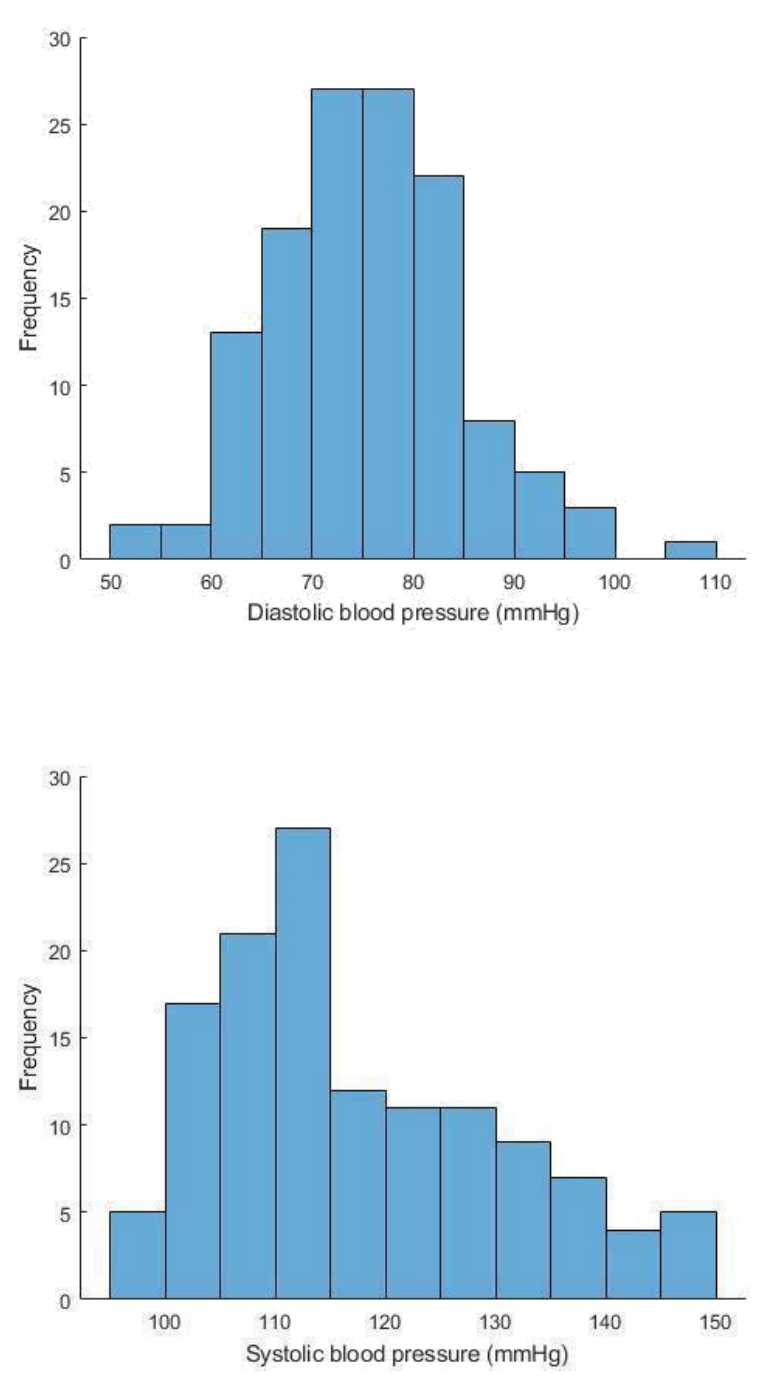

Fig. 2 Histogram of the collected blood pressure values: (a) diastolic blood pressure, (b) systolic blood pressure

The blood pressure distribution histograms of the remaining datasets are shown in Fig 2. Diastolic blood pressure ranges from $53 \mathrm{mmHg}$ to $108 \mathrm{mmHg}$ and systolic blood pressure from $97 \mathrm{mmHg}$ to $148 \mathrm{mmHg}$. Because the subjects were required to do different levels of exercise, the measured blood pressure range was relatively large, which could help to demonstrate the robustness of the blood pressure prediction results.

\subsection{Pre-processing}

Because the shortest duration for the recording of both ECG and PPG signals was 14 seconds, 10 seconds of the duration of each signal was selected, starting from the $5^{\text {th }}$ second, so as to eliminate the possible disturbance at the beginning of each recording.

For the ECG signal, the 4th Butterworth bandpass filter of 0.5-40 Hz was used to remove noise [17]. For the PPG signal, the 4th Chebyshev II bandpass filter of $0.5-10 \mathrm{~Hz}$ was adopted to improve the signal quality index (SQI) [18]. 
Consequently, the ECG and PPG signals were normalized, and one period were selected according to the maximal correlation value of the neighboring periods.

\subsection{Feature extraction}

Table 1 shows the summary of features extracted from the ECG and PPG signals. PTT was calculated as a time difference between the ECG and PPG peaks within one heartbeat. Except for the PTT and heart rate, the rest of features were extracted from both ECG and PPG signals. Hence, the number of these features was doubled, and there were 90 features from each dataset.

Table 1 Features extracted from ECG and PPG signals

\begin{tabular}{|c|c|c|}
\hline \multicolumn{2}{|l|}{ Feature name } & $\begin{array}{l}\text { Feature } \\
\text { numbers }\end{array}$ \\
\hline \multicolumn{2}{|c|}{ Pulse Transit time (PTT) } & 1 \\
\hline \multicolumn{2}{|c|}{$\begin{array}{l}\text { Autoregressive (AR) model coefficients of } \\
\text { order } 8\end{array}$} & 8 \\
\hline \multirow{2}{*}{$\begin{array}{l}\text { Multifractal } \\
\text { wavelet } \\
\text { leader }\end{array}$} & $\begin{array}{l}\text { Second cumulant of scaling } \\
\text { exponents }\end{array}$ & 1 \\
\hline & The range of Holder exponents & 1 \\
\hline \multicolumn{2}{|c|}{$\begin{array}{l}\text { Shannon Entropy (SE) values for the } \\
\text { maximal overlap discrete wavelet packet } \\
\text { transform at level } 5\end{array}$} & 32 \\
\hline \multirow{2}{*}{$\begin{array}{l}\text { Hjorth } \\
\text { Parameters } \\
\end{array}$} & \begin{tabular}{l|l} 
Signal mobility \\
\cline { 2 - 2 }
\end{tabular} & 1 \\
\hline & Signal complexity & 1 \\
\hline \multicolumn{2}{|l|}{ Heart rate } & 1 \\
\hline
\end{tabular}

In signal processing, an autoregressive (AR) model can represent a type of random process and it indicates that the output variable depends linearly on its own previous values (each having a coefficient) and on a stochastic term. Eight orders of coefficients were calculated and used as features [19]. Multifractal wavelet leader estimates of the second cumulant of the scaling exponents and the range of Holder exponents, or singularity spectrum were used as features from ECG and PPG signals [20]. Shannon entropy (SE) values for the maximal overlap discrete wavelet packet transform at level 5 [21] and signal mobility and signal complexity were calculated by using the Hjorth parameters method [13].

\subsection{Machine learning}

In this study, two machine learning models were chosen to predict the blood pressure values: Lasso regression and support vector machine (SVM). The aim of this study is to investigate whether the inclusion of ECG features can improve the accuracy of prediction of the continuous, noninvasive blood pressure. After the feature extraction, 129 groups of datasets were divided into two sub-datasets. $70 \%$ of the datasets were used for training the machine learning models while $30 \%$ of the datasets were for the models' testing. Models were established to estimate systolic blood pressure and diastolic blood pressure separately, both with and without ECG features. The mean absolute error (MAE) and standard deviation (STD) of estimation errors were used to evaluate the models after training.

\section{Results}

The results from two different regression approaches described above are presented in Tables 2 and 3. Table 2 demonstrates the prediction of the diastolic blood pressures, including and excluding ECG signal features. In both the Lasso regression and the SVM, the inclusion of the ECG features can improve the performance of the models, although the improvement is not significant. Furthermore, comparing the Lasso regression with the SVM, it can be seen that the SVM has smaller MAE and STD values.

Table 2 The predicated results of diastolic blood pressure (DBP) from Lasso and SVM

\begin{tabular}{|c|c|c|c|c|}
\hline \multirow{2}{*}{ DBP } & \multicolumn{2}{|c|}{ Lasso } & \multicolumn{2}{c|}{ SVM } \\
\cline { 2 - 5 } & MAE & STD & MAE & STD \\
\hline $\begin{array}{c}\text { With } \\
\text { ECG }\end{array}$ & 5.73 & 7.28 & 5.22 & 6.65 \\
\hline $\begin{array}{c}\text { Without } \\
\text { ECG }\end{array}$ & 5.79 & 7.40 & 5.48 & 6.69 \\
\hline \multicolumn{4}{|c|}{ The unit in this table is mmHg } \\
\hline
\end{tabular}

From Table 3, it is evident that inclusion of ECG features leads to a slightly better predictive performance for systolic blood pressure compared with the case of excluding ECG features. Overall, the performance of the SVM is better than that of the Lasso regression.

Table 3 The predicated results of systolic blood pressure (SBP) from Lasso and SVM

\begin{tabular}{|c|c|c|c|c|}
\hline \multirow{2}{*}{ SBP } & \multicolumn{2}{|c|}{ Lasso } & \multicolumn{2}{c|}{ SVM } \\
\cline { 2 - 5 } & MAE & STD & MAE & STD \\
\hline $\begin{array}{c}\text { With } \\
\text { ECG }\end{array}$ & 8.94 & 11.0 & 7.33 & 9.5 \\
\hline $\begin{array}{c}\text { Without } \\
\text { ECG }\end{array}$ & 9.4 & 11.6 & 9.75 & 12.3 \\
\hline \multicolumn{3}{|c|}{ The unit in this table is mmHg } \\
\hline
\end{tabular}

Other cuff-less blood pressure estimation designs in literature, such as using PTT alone to predict blood pressure, suffer from different drawbacks such as requiring calibration for each person. Table 4 gives continuous, cuff-less blood pressure measurement method results obtained by Kachuee et al. in 2015 [16]. In their research, blood pressure was estimated by extracting several morphological features from the PPG signal, and then applying signal processing and machine learning algorithms. Features from ECG signals were not included and the results were shown in Table 4 , which can be compared with the results from this study listed in Table 2 and 3. The overall results of this study are much more accurate than these obtained by Kachuee et al. Here the criteria of comparison is based on the values of deviations of the predicted results from the results that meet the Association for the Advancement of Medical Instrumentation (AAMI) (MAE is $\pm 5 \mathrm{mmHg}$ and STD within $8 \mathrm{mmHg}$ ) and British Hypertension Society (BHS) standards [22]. As a result, the inclusion of ECG signal can indeed improve the prediction accuracy of blood pressure. 
Table 4 The blood pressure estimation results from Kachuee et al. [16]

\begin{tabular}{|c|c|c|c|c|}
\hline \multirow{2}{*}{ Algorithm } & \multicolumn{2}{|c|}{ DBP } & \multicolumn{2}{c|}{ SBP } \\
\cline { 2 - 5 } & MAE & STD & MAE & STD \\
\hline Linear & 7.24 & 9.23 & 14.73 & 18.47 \\
\hline SVM & 6.34 & 8.45 & 12.38 & 16.17 \\
\hline \multicolumn{4}{|c|}{ The unit in this table is mmHg } \\
\hline
\end{tabular}

\section{Conclusion}

With widespread and growing hypertension, developing a cuff-less and continuous blood pressure measurement system is vital to human health. The proposed method in this paper establishes a cuff-less, continuous, and non-invasive blood pressure measurement method which makes this goal achievable. A novel method has been established in this study to carry out continuous and cuff-less blood pressure estimation with features extracted from both ECG and PPG signals. This has been validated by the use of two machine learning models, the Lasso regression and the SVM. The results are compared with other blood pressure prediction work reported in literature. In conclusion, the inclusion of ECG features can indeed improve the performance of blood pressure estimation. Further, the frequency domain features extracted in this study are different from these used in earlier studies $[14,23,24]$, which are generally based on morphological or time domain parameters. Additional analysis of these frequency features may lead to new understandings in cardiovascular health.

\section{Acknowledgements}

The authors acknowledge the financial support from the International Doctoral Innovation Centre, Ningbo Education Bureau, Ningbo Science and Technology Bureau, and the University of Nottingham. This work was also supported by the UK Engineering and Physical Sciences Research Council [grant numbers EP/G037345/1 and EP/L016362/1] and Optics and Photonics Research Group in the University of Nottingham.

\section{References}

[1] Agarwal, R., Jennifer E., Hecht, T.J.W., et al.: 'Role of home blood pressure monitoring in overcoming therapeutic inertia and improving hypertension control: a systematic review and meta-analysis', Hypertension, 2011, 57, (1), pp. 29-38

[2] Burt, V.L., Whelton, P., Roccella, E.J., et al.: 'Prevalence of hypertension in the US adult population. Results from the Third National Health and Nutrition Examination Survey, 1988-1991', Hypertension, 1995, 25, (3), pp. 305-313

[3] 'Affordable technology : blood pressure measuring devices for low resource settings'. (Geneva : World Health Organization, 2005)

[4] Zheng, Y.-L., Ding, X.-R., Poon, C.C.Y., et al.: 'Unobtrusive Sensing and Wearable Devices for Health
Informatics', IEEE Transactions on Biomedical Engineering, 2014, 61, (5), pp. 1538-1554

[5] 'A global brief on hypertension : silent killer, global public health crisis: World Health Day 2013', http://apps.who.int/iris/handle/10665/79059, accessed November 2018

[6] Chung, E., Chen, G., Alexander, B., et al.: 'Non-invasive continuous blood pressure monitoring: a review of current applications', Frontiers of Medicine, 2013, 7, (1), pp. 91-101 [7] Peter, L., Noury, N., Cerny, M.: 'A review of methods for non-invasive and continuous blood pressure monitoring: Pulse transit time method is promising?', IRBM, 2014, 35, (5), pp. 271-282

[8] Sharma, M., Barbosa, K., Ho, V., et al.: 'Cuff-Less and Continuous Blood Pressure Monitoring: A Methodological Review', Technologies, 2017, 5, (2), p. 21

[9] Yang, S., Zhang, Y., Morgan, S., et al.: 'Cuff-less blood pressure measurement using fingertip photoplethysmogram signals and physiological characteristics', Proc. Optics in Health Care and Biomedical Optics VIII, SPIE,

October 2018, p. 116

[10] Wang, L., Zhou, W., Xing, Y., et al.: ‘A Novel Neural Network Model for Blood Pressure Estimation Using Photoplethesmography without Electrocardiogram', Journal of Healthcare Engineering, 2018, 2018, pp. 1-9

[11] Addison, P.S.: 'Slope Transit Time (STT): A Pulse Transit Time Proxy requiring Only a Single Signal Fiducial Point', IEEE Transactions on Biomedical Engineering, 2016, 63, (11), pp. 2441-2444

[12] Watanabe, N., Bando, Y.K., Kawachi, T., et al.: 'Development and Validation of a Novel Cuff-Less Blood Pressure Monitoring Device', JACC: Basic to Translational Science, 2017, 2, (6), pp. 631-642 [13] Simjanoska, M., Gjoreski, M., Gams, M., et al.: 'NonInvasive Blood Pressure Estimation from ECG Using Machine Learning Techniques', Sensors, 2018, 18, (4), p. 1160

[14] Wu, C.-M., Chuang, C.Y., Chen, Y.-J., et al.: 'A new estimate technology of non-invasive continuous blood pressure measurement based on electrocardiograph', Advances in Mechanical Engineering, 2016, 8, (6), pp. 1-8 [15] Estrada, G. M., Mendoza, L.E., Molina, V.:

'Relationship of blood pressure with the electrical signal of the heart using signal processing', TECCIENCIA, 2014, 9, (17), pp. 9-16

[16] Kachuee, M., Kiani, M.M., Mohammadzade, H., et al.: 'Cuff-less high-accuracy calibration-free blood pressure estimation using pulse transit time', Proc. 2015 IEEE International Symposium on Circuits and Systems (ISCAS), 2015, pp. 1006-1009

[17] Shin, W., Cha, Y.D., Yoon, G.: 'ECG/PPG integer signal processing for a ubiquitous health monitoring system',

J Med Syst, 2010, 34, (5), pp. 891-898

[18] Liang, Y., Elgendi, M., Chen, Z., et al.: 'An optimal filter for short photoplethysmogram signals', Scientific Data, 2018, 5, p. 180076

[19] Qibin Zhao, Liqing Zhang: 'ECG Feature Extraction and Classification Using Wavelet Transform and Support Vector Machines', Proc. 2005 International Conference on Neural Networks and Brain, IEEE, 2005, pp. 1089-1092 
[20] Leonarduzzi, R.F., Schlotthauer, G., Torres, M.E.:

'Wavelet leader based multifractal analysis of heart rate variability during myocardial ischaemia', Proc. 32nd Annual International Conference of the IEEE Engineering in Medicine and Biology Society (EMBC), IEEE, 2010, pp. 110-113

[21] Li, T., Zhou, M.: 'ECG Classification Using Wavelet Packet Entropy and Random Forests', Entropy, 2016, 18, (8), p. 285

[22] Jones, D.W., Hall, J.E.: 'The national high blood pressure education program: thirty years and counting', Hypertension, 2002, 39, (5), pp. 941-942

[23] Kavsaoğlu, A. R., Polat, K., Bozkurt, M. R.: ‘A novel feature ranking algorithm for biometric recognition with PPG signals', Computers in Biology and Medicine, 2014, 49, pp. $1-14$

[24] Kachuee, M., Kiani, M.M., Mohammadzade, H., et al.: 'Cuffless Blood Pressure Estimation Algorithms for Continuous Health-Care Monitoring', IEEE Transactions on Biomedical Engineering, 2017, 64, (4), pp. 859-869 